\title{
Self-control and its influence on global/local processing: An investigation of the role of frontal alpha asymmetry and dispositional approach tendencies
}

\author{
Brent Pitchford ${ }^{1} \cdot$ Karen M. Arnell ${ }^{1}$
}

Published online: 23 October 2018

(C) The Psychonomic Society, Inc. 2018

\begin{abstract}
People often inhibit or override their dominant response tendencies in order to complete tasks successfully. Exerting such selfcontrol has been shown to influence attentional breadth differently depending on approach-motivated tendencies, as indexed by individuals' behavioral activation system (BAS) scores. Approach motivation and attentional breadth have previously been associated with frontal alpha asymmetry (i.e., lateralized cortical activity in the frontal regions) where greater left-frontal activation is associated with greater approach motivation and reduced attentional breadth. The process model of self-control posits that exercising self-control leads to a subsequent increase in approach behavior in high BAS individuals, and this could be due to a shift towards left-hemisphere-frontal processing. This was the first study to examine both frontal asymmetry and attentional breadth before and after exercising self-control in low and high BAS individuals. Greater BAS, and greater difficulty exercising self-control, both positively related to more narrowed attentional breadth after completing the manipulation relative to before, but only after exercising self-control. However, breadth of attention and changes in attentional breadth were unrelated to frontal asymmetry, suggesting that the influence of self-control on individuals' attentional breadth was not due to changes in frontal activation patterns.
\end{abstract}

Keywords Attention $\cdot$ Electrophysiology $\cdot$ Cognitive $\cdot$ Attentional control

\section{Seeing the forest or the trees: Global/local processing}

Visual stimuli can often be perceived in their entirety or as smaller elements. For example, the hierarchical Navon letter (Navon, 1977) shown in Fig. 1 can be seen as a large F (global) or as several smaller Ts (local). An individual's attentional breadth is measured through their inclination to attend to the local or global level; a local bias reflects greater focus on the local level and indicates narrowed attention, whereas a global bias reflects greater focus on the global level and indicates broad attention. For example, individuals with a global bias may be faster to detect a target letter at the global level than at the local level (e.g., Gable \& Harmon-Jones, 2008), may show greater interference from the global level when needing to attend to the local level (Dale \& Arnell, 2013;

Karen M. Arnell

karnell@brocku.ca

1 Department of Psychology, Brock University, 1812 Sir Isaac Brock Way, St. Catharines, ON L2S 3A1, Canada
Navon, 1977), and may be more likely to report seeing the global level (forest) over the local level (trees) such as when they identify a triangle made of squares as more similar to a triangle made of triangles than to a square made of squares in the hierarchical shape task (e.g., Dale \& Arnell, 2013; Fredrickson \& Branigan, 2005; Kimchi \& Palmer, 1982).

There are reliable individual differences in global/local biases when tasks and stimuli are kept constant. Some individuals show a bias toward focusing on the local level, while others show a bias toward focusing on the global level, and these biases have been found to be relatively stable over at least 10 days in multiple global/local tasks (Dale \& Arnell, 2013). However, changes in stimulus parameters, such as visual angle (Kinchla \& Wolfe, 1979), time of exposure (Paquet \& Merikle, 1984), or the aspect ratio (Kimchi, 1992), have also been found to influence an individual's global/local bias, as have situational or state manipulations. For example, inducing affective mood states has been shown to influence attentional breadth (Fredrickson \& Branigan, 2005; Gable-and Harmon-Jones, 2008) where positive mood states that are low in motivational intensity (e.g., satisfaction, serenity) increase global bias, whereas positive mood states that are high 


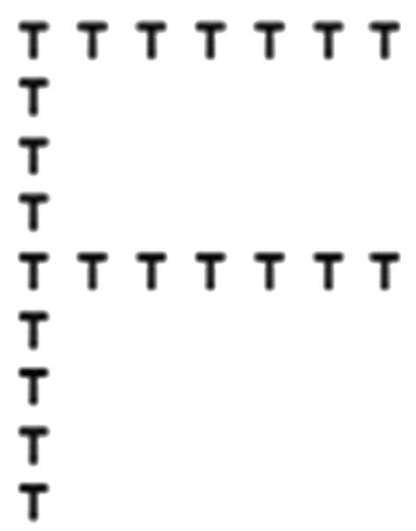

Fig. 1 Example of an incongruent Navon letter stimulus (e.g., Navon, 1977)

in motivational intensity (e.g., desire, engagement) decrease global bias.

For the sake of survival, it is crucial to approach stimuli that are desirable, such as food or potential mates. To demonstrate that approach-motivated positive affect could narrow attentional breadth, Gable and Harmon-Jones (2008) used Navon (1977) letters to measure attentional breadth, both after viewing high approach-motivating, positively-valenced images (i.e., desserts) and neutral images (i.e., rocks). They found that individuals were faster at indicating the smaller, or local, elements and slower at indicating the global elements, after viewing pictures of desserts relative to the neutral images, and this effect increased when individuals were led to believe that they would get to consume one of the desserts at the end of the study. They suggested that attentional breadth decreases so that individuals can focus on objects they desire when they are in an approach-motivated positive affective state, which can help aid in goal pursuit (Gable \& Harmon-Jones, 2011). Further evidence for this hypothesis was provided in that people reporting higher approach motivation in everyday life - as indexed by their behavioral activation system (BAS) (Carver \& White, 1994) scale scores - showed a greater local bias (narrower attention) after viewing pictures of desserts relative to those that reported lower approach motivation. These results suggest that approach-motivated positive affect can narrow attention and that this effect is especially salient for individuals who have more naturally occurring approach motivation in everyday life.

Multiple studies have found that motivation is reflected asymmetrically in the prefrontal areas of the cortex where greater left prefrontal activity is associated with greater approach motivation, and greater right frontal activity is associated with greater withdrawal motivation (for review, see Spielberg, Stewart, Levin, Miller, \& Heller, 2010). For example, Harmon-Jones and Allen (1998) found that individuals' dispositional anger (a high approach emotion) was associated with greater left prefrontal activity at rest despite anger having a negative valence. Furthermore, greater left prefrontal activity at rest has also been related to increased responding to reward-related cues (Hughes, Yates, Morton, \& Smillie, 2014; Pizzagalli, Sherwood, Henriques, \& Davidson, 2005), greater optimism (De Pascalis, Cozzuto, Caprara \& Alessandri, 2013) and reduced sensitivity to negative outcomes (Nash, Inzlicht, \& McGregor, 2012). Greater right prefrontal activity at rest has been related to greater stress response (Düsing, Tops, Radtke, Kuhl, \& Quirin, 2016; Quaedflieg, Meyer, Smulders, \& Smeets, 2015; Shields \& Moons, 2016), increased activation of the supervisory control system (Gable, Mechin, Hicks, \& Adams, 2015), biases to threat-especially under stress (Grimshaw, Foster, \& Corballis, 2014; Pérez-Edgar, Kujawa, Nelson, Cole, \& Zapp, 2013), and risk of psychopathology such as depression (for review see Coan \& Allen, 2004).

Indeed, manipulations of frontal asymmetry can have a causal role on individuals' approach motivation (for a review, see Kelley, Hortensius, Schutter, \& Harmon-Jones, 2017). For example, increasing left frontal activity can cause people to make riskier decisions (Fecteau et al., 2007), while increasing right frontal activity can cause decreased food cravings (Fregni et al., 2008) — results that are consistent with left frontal activations association with approach, and right frontal activations association with withdrawal. Price and HarmonJones (2010) also provided experimental evidence for the link between approach motivation and left hemisphere frontal activation by showing greater left versus right hemisphere frontal cortical activation when participants leaned forward with their arms extending forward (a high approach posture) compared with when they reclined backward (a low approach posture). Importantly, frontal asymmetry has also been related to individuals' BAS scores, as higher BAS scores have been associated with larger relative left resting frontal activity (Coan \& Allen, 2003; Harmon-Jones \& Allen, 1997), although this relationship has not always been found (Boksem, Kostermans, Tops, \& De Cremer, 2012; Neal \& Gable, 2017; Wacker, Chavanon, \& Stemmler, 2010).

There is experimental evidence that increasing relative left activity can cause narrower attentional breadth, while increasing relative right activity can cause increased attentional breadth. Gable, Poole, and Cook (2013) asked participants to squeeze a small rubber ball in either their left or right hand in order to increase contralateral hemispheric activity. They found that these hand contractions resulted in greater contralateral activation, whereby squeezing the ball in one's right hand resulted in greater left hemispheric activation and vice versa. After squeezing the rubber ball, participants then completed the Navon letter task to assess their attentional scope. Participants that had shown greater left hemispheric activation by squeezing the rubber ball in their right hand were found to have a more narrowed attentional scope (i.e., they were more locally biased), while participants that had squeezed the rubber ball in their left hand showed a broader attentional scope 
(i.e., greater global bias). These results suggest that there is a causal link between relative asymmetrical activity and attentional breadth, whereby greater left relative activity results in narrower attentional scope and greater right relative activity results in broadened attentional scope.

Harmon-Jones and Gable (2009) examined relative left frontal activity while people viewed either a dessert image or a neutral image prior to each Navon letter. Their results showed a local bias on trials following a dessert picture, but a global bias on trials following a neutral picture. Furthermore, individual differences in left frontal activity while viewing dessert images predicted faster local target reaction times, even when controlling for local reaction times after viewing neutral images. Additionally, the strength of this association increased with the number of hours since the participant had eaten.

Exercising self-control can also potentially influence frontal asymmetry (Schmeichel, Crowell, \& Harmon-Jones, 2016). Self-control is defined as "the aspect of inhibitory control that involves resisting temptations and not acting impulsively or prematurely" (Diamond, 2013, p. 137). Sometimes people must inhibit prepotent or tempting responses in order to make more appropriate responses. An example in everyday life would be if a person had to inhibit the temptation to eat some cake, which may be much more rewarding in the shortterm, to better reach their potentially more rewarding longterm goals such as being healthier.

There have been attempts in recent years to determine precisely what occurs during regulatory failures. According to the process model, self-control does not rely on a limited resource, per se, but rather, exercising self-control by consciously and effortfully inhibiting predominant response tendencies may lead to a shift in people's motivational orientation (Inzlicht \& Schmeichel, 2012). Engaging in self-control is taxing, effortful, and often aversive (Botvinick, 2007), and people may switch from working on "have-to" goals towards indulging in their "want-to" goals (Inzlicht \& Schmeichel, 2012). Therefore, according to the process model, greater motivation toward internally gratifying, enjoyable and meaningful activities after completing self-control may lead to an increase in approach behavior following self-control (Schmeichel, Harmon-Jones, \& Harmon-Jones, 2010).

There is evidence to suggest that individuals do experience greater approach motivation after exercising self-control. For example, participants showed increased betting on a lowstakes gambling game, regardless of relative outcomes, as well as facilitated perception of reward-related symbols (i.e., dollar signs) after exercising self-control (Schmeichel et al., 2010). Also, individuals with higher BAS scores demonstrated greater optimism about future health outcomes after engaging in self-control (Crowell, Kelley, \& Schmeichel, 2014). Exercising self-control also resulted in greater subsequent activity in areas of the brain that are responsible for coding reward (Wagner, Altman, Boswell, Kelley, \& Heatherton, 2013) as well as increased desire (Hofmann, Vohs, \& Baumeister, 2012).

Importantly, self-control can also influence frontal asymmetry whereby exercising self-control can result in greater left relative frontal activity - a proposed marker of approach motivation - especially for those who self-report high approach motivation in everyday life. In a recent study, Schmeichel et al. (2016) asked participants to complete a writing task where they were instructed to write about a trip that they had recently taken. Half of them were asked to exercise self-control by refraining from using the letters "a" or " $n$," while the other half were given no further instructions (i.e., the control group). Afterward, they were asked to view positive, negative, and neutral images while EEG was recorded, and then complete the BIS/BAS questionnaire to measure naturally occurring approach motivation. Completing the controlled writing task (i.e., inhibiting the use of the two letters to elicit self-control), but not the free-writing task, increased relative left frontal activity when viewing positive images for individuals with high BAS-BIS scores, but not for those with low BAS-BIS scores.

Exercising self-control has been hypothesized to increase approach motivation and has been shown to increase left frontal activation, at least for individuals high in trait approach motivation (Schmeichel et al., 2016). Both approach motivation and left frontal activation have been implicated in a local processing bias (e.g., Gable et al., 2013; Harmon-Jones \& Gable, 2009), suggesting that exercising self-control could promote reduced attentional breadth. Crowell et al. (2014) had participants complete the BIS/BAS questionnaire after writing about a recent trip they had taken, either without constraints (control condition) or without using the letters " $a$ " or " $\mathrm{n}$ " (self-control condition), and completing the hierarchical shape task to measure their attentional breadth (Kimchi \& Palmer, 1982). Participants reporting greater trait approach motivation made more global selections, but only after completing the more taxing, self-control task. This suggests that exercising self-control broadened their attention, which the authors suggested was an indication of greater approach motivation following self-control. However, this finding and conclusion are contrary to what one would expect based on the association between self-control, approach motivation, and local bias as outlined above.

\section{Experiment 1}

The aim of the first experiment was to replicate Crowell et al.' (2014) finding that attentional breadth could be influenced by having individuals exercise self-control for a brief period of time, and that this relationship would be moderated by individuals' self-reported approach motivation as indicated by their BAS scores. Participants completed the BIS/BAS scales 
and performed a global/local task to measure attentional breadth both before and after a 5-minute Stroop task requiring high self-control (incongruent Stroop trials) or low selfcontrol (congruent Stroop trials). Because local bias and approach motivation have both been associated with increases in left hemisphere activation, we predicted that attentional breadth would become narrower following self-control, but only for those with higher approach-motivated tendencies.

\section{Method}

\section{Participants}

The participants were 63 Brock University undergraduate students (12 males and 51 females), recruited through an online participant recruiting system. They all received partial course credit for their participation in the experiment. Participants ranged in age from 17 to 25 years, with a mean age of 19.72 years $(S D=1.86)$. All participants reported English being their first language and had normal or corrected-to-normal vision. None of the participants reported any perceptual or cognitive impairment.

BIS/BAS The Behavioral Activation System and Behavioral Inhibition System (BIS/BAS) scales (Carver \& White, 1994) were used to measure individuals' dispositional motivational systems. The BAS scale consists of 13 statements, while the BIS scale consists of seven statements. Participants completed a computerized version of the questionnaire, with items from both scales presented in random order. They were asked to report on a scale from 1 (strongly disagree) to 4 (strongly agree) whether the behavior described in each item was like them. Individuals' BAS scores were calculated by summing individuals' responses to all 13 items such that the minimum possible score was 13 and the maximum possible score was 52 .

\section{Computerized tasks}

Navon letter task In the Navon letter task (Navon, 1977), participants were presented with large (global) letter stimuli that were composed of smaller (local) letter stimuli (see Fig. 1 for an example). Smaller letters had a visual angle of $0.19^{\circ}$ (height) by $0.19^{\circ}$ (width), while the larger letters had a visual angle of $3.82^{\circ}$ by $2.39^{\circ}$. Each letter was centrally presented on a white-background screen, until the response was made, and was preceded by a fixation cross for $500 \mathrm{~ms}$ and followed by a blank screen for 1,000 ms. The Navon letters were always incongruent in that the global-level letter was different from the local-level letters. The letters $T$ and $H$ were designated as targets, and the participant was instructed to press the key matching the identity of the target letter as quickly and accurately as possible. On half of the trials, a target letter (i.e., $T$ or $H$ ) was presented at the local level and a distractor (i.e., $F$ or $L$ ) was presented at the global level. On the other half of trials, a target letter was presented at the global level and a nontarget letter was presented at the local level. All combinations of target letters, distractor letters, and target levels were equally likely and presented randomly trial to trial. Participants completed 64 trials of the Navon task before the Stroop task, and 64 trials immediately after the Stroop task (Stroop, 1935).

Stroop color task In the Stroop color task (Stroop, 1935), a color word, presented in 18-point Courier New font, was presented in the center of a white computer screen until a response was made. This was preceded by a 500 -ms fixation cross and followed by a 1,000-ms blank screen after the response. Four colors (red, blue, green, and orange) were used for the font colors and words. In the congruent condition, font colors matched the word meanings (e.g., the word "BLUE" in blue font). In the incongruent condition, font colors did not match the word meanings (e.g., the word "BLUE" in red font). Participants were randomly assigned to complete 5 minutes of either incongruent Stroop trials or congruent Stroop trials. ${ }^{1}$ All participants were instructed to indicate the font (i.e., ink) color of the word by pressing one of the $A, S$, $K, L$ keys, with corresponding color stickers, as quickly and accurately as they could.

ProcedureAll questionnaire items, Stroop, and Navon stimuli, were presented on a 17-inch CRT monitor and controlled by E-Prime software running on a Dell desktop computer. Responses were made using the computer keyboard or mouse while participants viewed the screen unrestrained from approximately 60 centimeters. Responses were timed using E-Prime software (Psychology Software Tools, Pittsburgh, PA, USA). All participants performed the tasks alone in a test cubicle.

After completing the questionnaires, ${ }^{2}$ participants completed the first block of the Navon letter task, then the Stroop task for 5 minutes, regardless of how many trials they had completed, followed by a second block of Navon trials. Afterwards, they were asked to report on a scale of 1 (not difficult at all) to 4 (extremely difficult), how challenging they found the Stroop task. The total time that it took to complete the computerized tasks following the questionnaires was approximately 11 minutes.

\section{Results}

\section{BIS/BAS}

Similar to past research (e.g., Gable \& Harmon-Jones, 2008; Spielberg et al., 2012), BAS scores were used as self-reported

\footnotetext{
${ }^{1}$ The number of Stroop trials completed by participants in 5 minutes did not significantly relate to any dependent measures.

2 Participants also completed three additional computerized questionnaires that did not measure approach or withdrawal motivation. These measures were not the focus of this study and will not be discussed further here. In total, the questionnaires were completed in less than 30 minutes.
} 
indicators of individuals' approach motivation. The mean BAS score was 38.73 (range: 13-52), with a standard deviation of 4.84, making it comparable to previous research (e.g., Carver \& White, 1994; Harmon-Jones \& Allen, 1997), and showing a good range of self-reported approach motivation by individuals within our sample.

\section{Stroop color task}

Stroop response times (RTs) were extracted for all correct trials $\left(M_{\text {reject }}=3.66 \%, S D_{\text {reject }}=2.97 \%\right)$. One participant's data were not included in the analyses due to the absence of any correct Stroop trials, suggesting they did not complete the manipulation as instructed. RTs from the remaining participants $(N=62)$ were subjected to a two-stage recursive outlier elimination procedure, where RTs were removed if they were greater than or less than two standard deviations from the mean for each participant $\left(M_{\text {reject }}=7.91 \%, S D_{\text {reject }}=4.35 \%\right)$.

As expected, participants in the incongruent Stroop condition had significantly longer Stroop RTs $(M=775 \mathrm{~ms}, S D=$ $180 \mathrm{~ms}$ ) than did participants in the congruent Stroop condition $(M=606 \mathrm{~ms}, S D=137 \mathrm{~ms}), t(60)=4.15, p<.001, d=1.05$, and rated the Stroop task as significantly more difficult $(M=$ $2.65, S D=.80$ ) than participants in the congruent condition did $(M=2.06, S D=.93), t(60)=2.64, p=.01, d=.68$. There was no significant difference in accuracy for the incongruent $(M=$ $95.93 \%, S D=3.65 \%)$ and congruent $(M=96.86 \%, S D=$ $2.01 \%$ ) conditions, $t(46.64)=1.24, p=.22, d=.32$.

Navon letter task All correct global/local RTs that were less than 2,000 ms were extracted $\left(M_{\text {reject }}=2.50 \%, S D_{\text {reject }}=\right.$ $2.93 \%)$. These were subjected to a two-stage recursive outlier elimination procedure, where RTs were removed if they were greater than or less than two standard deviations from the mean for each combination of participant, level (global, local), and time (pre-Stroop Navon, post-Stroop Navon) $\left(M_{\text {reject }}=\right.$ $\left.7.71 \%, S D_{\text {reject }}=2.03 \%\right)$. Participants who had local and/or global RTs that were greater than or less than three standard deviations away from the sample mean for each combination of level and time were excluded from analysis, resulting in the exclusion of four participants. Similar to previous research, a difference score was calculated (local RT - global RT), to represent attentional breadth (e.g., Juergensen \& Demaree, 2015). ${ }^{3}$ Attentional breadth change values were calculated as post-Stroop Navon attentional breadth minus pre-Stroop

\footnotetext{
$\overline{3}$ Although a difference score was used for analyses, standardized residuals (local RTs controlling for global RTs) could also have been used for analyses instead of difference scores. Indeed, the standardized residuals were correlated with the difference scores .88 for the pre-Stroop Navon attentional breadth measure, .89 for the post-Stroop Navon attentional breadth measure, and $r=$ $.78, p<.001$ for the pre-Stroop to post-Stroop Navon change in attentional breadth. Findings from this Experiment remained the same when using either difference scores or standardized residuals as dependent measures.
}

Navon attentional breadth, such that values greater than zero represented a shift toward broader attention following Stroop, and values less than zero represented a shift toward more narrowed attention following Stroop, relative to baseline (i.e., pre-Stoop).

There was a significant positive relationship between attentional breadth before Stroop and after Stroop, $r=.48, p<.001$, which is consistent with previous research (e.g., Dale \& Arnell, 2014) suggesting that there are reliable individual differences in global/local processing (see Table 1 for means and standard deviations). Note that both pre-Stroop and post-Stroop Navon blocks had mean attentional breadth scores close to zero, which indicates that there was no global or local bias in the sample overall. This is consistent with the fact that the Navon letters used here were created by Dale and Arnell $(2011,2013)$ to be global/local neutral across a sample (by adjusting the density and size of the local elements relative to the global element) so that an individual's natural global or local bias would not be dominated by the bias inherent in the stimuli.

\section{Changes in attentional breadth as moderated by trait approach motivation}

To investigate whether individual differences in naturally occurring approach motivation predicted changes in individuals' attentional breadth (i.e., attentional breadth after Stroop minus attentional breadth before), we regressed changes in attentional breadth on trait BAS scores (centered), Stroop condition (congruent or incongruent; coded as 0 and 1), and the condition by BAS interaction (see Fig. 2 for these results). There was a significant interaction between the self-control condition and BAS scores, $b=-53.30, t(54)=2.36, p=.02$. Simple slope analysis revealed that, in the incongruent Stroop condition, greater BAS scores predicted reduced attentional breadth pre-Stroop to post-Stroop Navon, $b=-37.82, t(54)=2.41, p=$ .02. In contrast, in the congruent Stroop condition, BAS scores did not predict changes in attentional breadth preStroop to post-Stroop Navon, $b=15.47, t(54)=.95, p=.35$. Furthermore, simple slopes showed that those high in BAS $(+1 S D)$ showed a significant effect of Stroop condition $b=$ $-84.31, t(54)=-2.58, p=.01$, that was not observed for those low in BAS $(-1 S D), b=25.31, t(54)=.77, p=.44$.

Table 1 Descriptive statistics for attentional breadth measures (ms) preStroop and post-Stroop, as well as the change in attentional breadth from pre-Stroop to post-Stroop $(N=58)$

\begin{tabular}{lllll}
\hline & Mean & $S D$ & Minimum & Maximum \\
\hline Pre-Stroop & -12 & 96 & -270 & 278 \\
Post-Stroop & 14 & 81 & -173 & 196 \\
Change & 25 & 92 & -130 & 259 \\
\hline
\end{tabular}




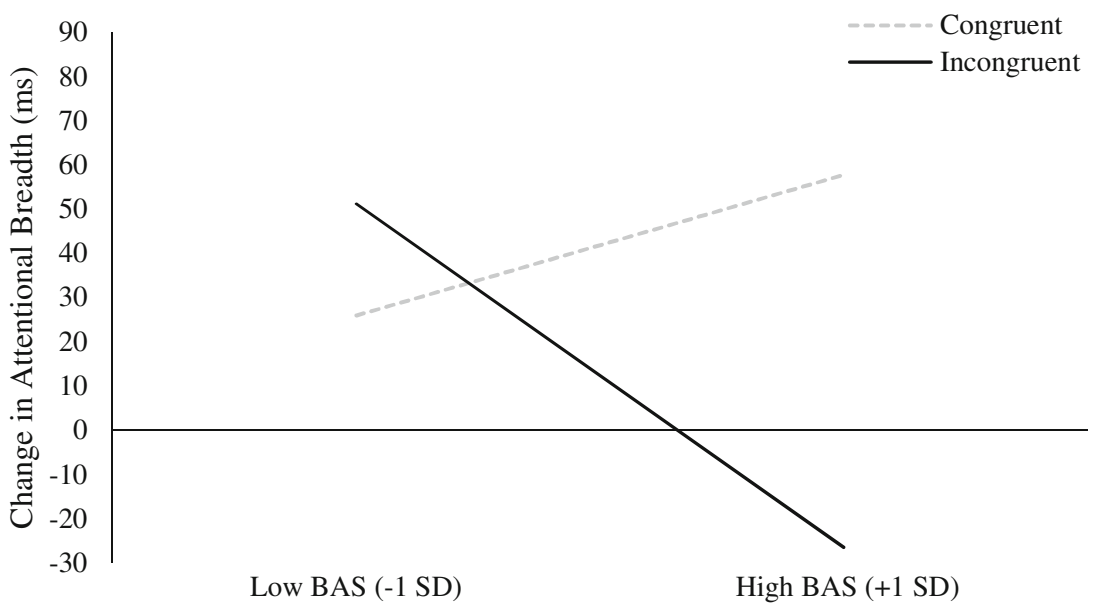

Fig. 2 Changes in attentional breadth (post-Stroop Navon attentional breadth - pre-Stroop Navon attentional breadth) for Navon stimuli as a function of trait approach motivation (BAS) and Stroop condition. Positive values for the change in attentional breadth represent a shift

toward a more global bias post-Stroop Navon relative to pre-Stroop Navon, whereas negative values represent a shift toward a more local bias post-Stroop Navon relative to pre-Stroop Navon

\section{Difficulty completing Stroop}

The degree to which individuals found the Stroop task to be difficult may have influenced their attentional breadth, especially if they were completing the incongruent Stroop condition where greater difficulty likely represented the need for, or use of, greater self-control. We first regressed changes in attentional breadth on Stroop condition and subjective ratings of Stroop difficulty (centered). As predicted, there was a significant interaction of the condition and difficulty ratings, $b=-59.37, t(54)=2.36, p=$ .02 (see Fig. 3). Simple slope analysis revealed that higher difficulty ratings significantly predicted attentional narrowing for participants in the incongruent Stroop condition, $b=-59.37$, $t(54)=2.02, p<.05$ but there was no relationship between difficulty ratings and attentional breadth for participants in the congruent Stroop condition, $b=19.83, t(54)=1.26, p=.21$.

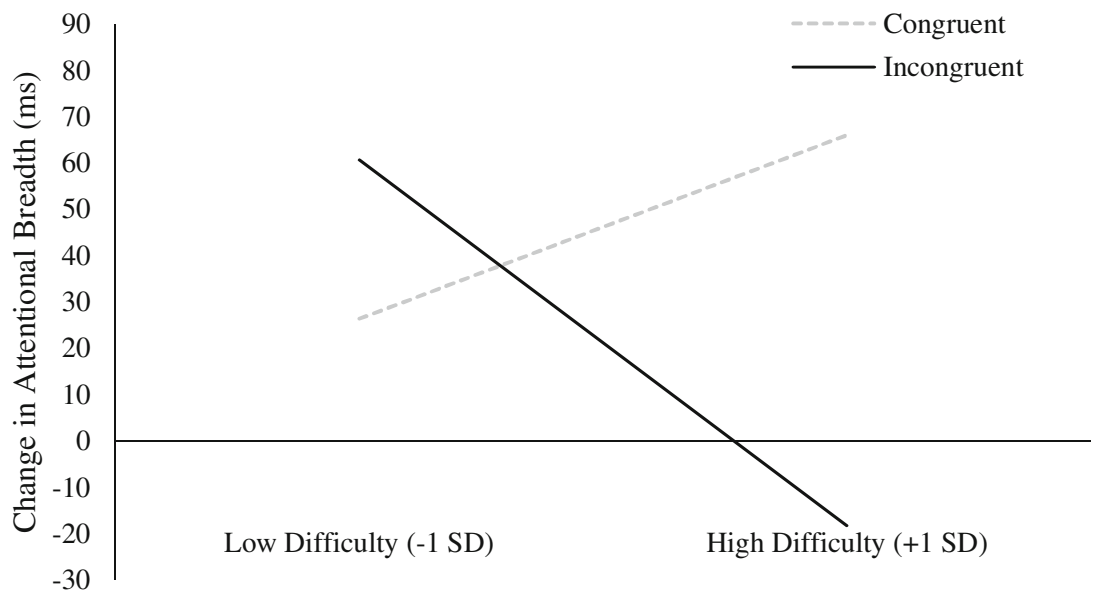

Fig. 3 Changes in attentional breadth (post-Stroop Navon attentional breadth - pre-Stroop Navon attentional breadth) for Navon stimuli as a function of individuals' difficulty rating for the Stroop task and Stroop condition. Positive values for the change in attentional breadth represent a
The results reported above show that, in the incongruent condition, both BAS and Stroop difficulty predicted reduced attentional breadth. Overall, when both self-reported difficulty (centered) and BAS (centered) were included as moderators of the relationship between Stroop condition and changes in attentional breadth, the Condition $\times$ BAS $\times$ Difficulty interaction was not significant, $b=.98, t(54)=.03, p=.98$. In the incongruent condition, BAS and Stroop difficulty were not related to each other, $r(30)=-.06, p>.76$. When BAS scores (centered), Stroop difficulty ratings (centered), and their interaction were entered as simultaneous predictors of attentional breadth change, the interaction was not significant, $b=$ $-10.46, t(24)=.53, p=.60$, and both BAS, $b=-39.07$, $t(24)=3.40, p<.01$, and Stroop difficulty, $b=-38.30, t(24)$ $=2.49, p=.02$, remained significant unique predictors of attentional breadth change over and above each other. shift toward a more global bias post-Stroop Navon relative to pre-Stroop Navon, whereas negative values represent a shift toward a more local bias post-Stroop Navon relative to pre-Stroop Navon 


\section{Discussion}

The results from Experiment 1 supported the hypothesis that exercising self-control can influence attentional breadth differently depending on self-reported approach motivation and that individuals high in BAS would show attentional narrowing after self-control. This pattern is consistent with previous research that has shown increased relative left frontal activity after exercising self-control for participants reporting higher levels of approach motivation (Schmeichel et al., 2016), as greater left frontal activity has been associated with greater local focus, and thus the increased relative left frontal activity after exercising self-control may relate to greater narrowed attention.

\section{Experiment 2}

Experiment 2 was a replication of the high self-control (incongruent Stroop) condition of Experiment 1, with added EEG measures. Resting and pre-Navon trial measures of frontal asymmetry were taken to test whether the change in frontal asymmetry following the exertion of self-control could account for the change in attentional breadth following the self-control manipulation, especially for high BAS participants. Based on findings in previous research (i.e., Schmeichel et al., 2016), and the process model of self-control (Inzlicht \& Schmeichel, 2012; Inzlicht, Schmeichel, \& Macrae, 2014), we hypothesized that exercising self-control would increase relative left frontal activity for individuals with high BAS, and that this change in frontal asymmetry would predict the change in attentional breadth. This hypothesis is based on the prediction that an anticipated increase in relative left frontal activity following the self-control task could be the mechanism as to how increased approachmotivated tendencies can predict more narrowed attentional breadth following self-control relative to before completing the self-control task. An association between the increase of relative left frontal activity following self-control and an increased narrowing of attention would provide support that asymmetrical frontal activity is indeed the mechanism.

\section{Method}

\section{Participants}

The participants were 71 Brock University undergraduate students recruited through an online participant recruiting system. They all received partial course credit for their participation in the experiment. All participants reported having proficient written English, normal or corrected-to-normal vision, and no history of neurological or cardiac conditions. Participants were all right-handed, able to discriminate colors, and were not taking psychoactive medications. Behavioral results were available for 63 participants: nine males and 54 females. These participants ranged in age from 18 to 48 years, with a mean age of 20.71 years $(S D=6.02)$. Behavioral results from eight participants were not included due to the following reasons: chance accuracy on at least one of the cognitive tasks (2), average Stroop RTs greater than three standard deviations above the sample mean (3), incomplete questionnaire data (2), an attentional breadth score that was greater than three deviations from the sample mean (1). The EEG data from 11 participants were not included due to the following reasons: incomplete data collection due to computer error (7), and the inability to acquire EEG signals with appropriate impedance levels (4). Therefore, of the total sample, both behavioral and EEG data were available for 56 participants: eight males and 48 females. Ages for these participants ranged from 18 to 48 years $(M=20.80$ years, $S D=6.23)$.

\section{Measures and procedure}

The BIS/BAS questionnaire, the Navon letter task and Stroop color task were identical to Experiment 1, with the exception that there was no congruent Stroop (control) condition implemented in Experiment 2. Recall that the results from Experiment 1 showed an association between BAS and changes in attentional breadth after self-control (the incongruent Stroop condition), but not after the control (congruent Stroop) condition. Therefore, only the incongruent Stroop condition was included in Experiment 2 so that the relationship between frontal asymmetry, BAS, and changes in attentional breadth could be explored in the condition of interest with increased power.The procedure for Experiment 2 was the same as Experiment 1, apart from the recording of EEG. After participants completed the questionnaires, and prior to the first block of Navon trials, the cap and electrodes were prepared and resting EEG was recorded as described below.

\section{EEG acquisition and preprocessing}

EEG was recorded continuously using 29 tin electrodes embedded in an Electro-Cap@ (Electro-Cap International Inc., Eaton, Ohio, USA) distributed according to the international 10-20 system. An electrode placed anterior to Fz was used as ground, while linked left and right earlobes were used as a reference. EEG data were amplified and acquired using a 32channel NeuroScan SynAmps and Neuroscan acquisition software (Compumedics USA, Charlotte, North Carolina) installed on a Dell desktop PC. EEG data were sampled online at a rate of $500 \mathrm{~Hz}$. Electrooculogram (EOG) electrodes were placed on the outer canthus of each eye and on the infra-orbital and supra-orbital regions of each eye to record horizontal and vertical eye movements, respectively. Impedance was maintained below 5 kiloohms for frontal sites of interest.

EEG data were analyzed off-line using EEGLAB Version 14.1.1b (Delorme \& Makeig, 2004) and custom routines written 
in MATLAB R2017a (The MathWorks, Natick, MA, USA). The data were band-pass filtered with the default EEGLAB filter (pop_eegfiltnew) excluding activity below $0.1 \mathrm{~Hz}$ and above $60 \mathrm{~Hz}$, and the default filter order (i.e., $2 \mathrm{~Hz}$ transition bandwidth). Artifacts (i.e., signals due to muscle movement, eye movements, and eye blinks) were manually removed using visual inspection and rejected from analysis. All activity \pm 100 microvolts in the vertical electrooculogram and horizontal electrooculogram was also rejected from analysis.

Resting EEG analysis EEG alpha asymmetry measures were gathered using eight 60 -second resting blocks that alternated between eyes open (EO) and eyes closed (EC) (i.e., either in one of the following orders: $\mathrm{COOCOCCO}$ or $\mathrm{OCCOCOOC}^{4}$ ). The EEG alpha asymmetry measures were calculated following recommended standard procedures (Allen, Coan, \& Nazarian, 2004). EC and EO data were separately epoched into 1.024second segments extracted through a hamming window where consecutive epochs overlapped by 50\%. An average of 170.23 epochs $(S D=102.20)$ comprised the EO data, and an average of 289.35 epochs $(S D=111.44)$ comprised the EC data. The number of accepted epochs did not relate to BAS, $p>.45$, or any attentional measures, $p \mathrm{~s}>.81$. Alpha power density $\left(\mathrm{V}^{2} /\right.$ $\mathrm{Hz} ; 8-13 \mathrm{~Hz}$ ) in each epoch was extracted using the pwelch() function and averaged across epochs of the same condition (i.e., EC and EO) separately for sites F3, F4, F7, and F8 (i.e., frontal electrode sites often used for calculating frontal asymmetry). Power values were log transformed to obtain normalized values. Creating an index of differential cortical activity by two sites on contralateral areas of the scalp (e.g., left and right of the midline) was done by subtracting the log-transformed power in the alpha band of the left sites from the right sites. Because greater alpha power is assumed to relate to inverse cortical activity (Allen et al., 2004), a greater value represented greater alpha activity in the right side and thus lesser cortical activity in the right side relative to the left. A medial frontal alpha asymmetrical index (log F4 - log F3) and a lateral alpha asymmetrical index $\left(\log\right.$ F8 - log F7) were determined ${ }^{5}$ for both $\mathrm{EO}$ and $\mathrm{EC}$ conditions, and then these values were averaged across EO and EC conditions. ${ }^{6}$ Finally, a resting frontal asymmetry score for each participant was determined by averaging their asymmetrical indices for the lateral and medial sites.

\footnotetext{
${ }^{4}$ An independent-samples $t$ test determined that the order of these blocks did not significantly influence resting frontal asymmetry, $t(44)=.07, p>.79$.

${ }^{5}$ Medial and lateral frontal asymmetry values were highly correlated, and the results reported here were also found when medial and lateral sites were examined separately.

${ }^{6}$ All asymmetry indices for EO data were highly correlated with asymmetry indices for $\mathrm{EC}$ data, $r \mathrm{~s}>.79$. Due to the strong relationships between asymmetrical measures of EO, EC data, as well as due to past findings suggesting that averaging asymmetry indices between $\mathrm{EO}$ and $\mathrm{EC}$ blocks produces better measures overall (Tomarken, Davidson, Wheeler, \& Kinney, 1992b), subsequent analyses were then performed using data collapsed across EO and EC blocks. The findings did not differ when using only EO or EC data.
}

Prestimulus EEG analysis Recall that each Navon letter was centrally presented until a response was made and was preceded by a fixation cross for $500 \mathrm{~ms}$ and a blank screen for $1,000 \mathrm{~ms}$. Epochs from $-1,024$ ms prior to onset of Navon letter stimuli were created to ensure consistent epoch length for both resting and task measures. An average of 55.15 seconds $(S D=11.22 \mathrm{~s})$ of data were available for pre-Stroop Navon analysis, while an average of 52.74 seconds $(S D=12.00 \mathrm{~s})$ of data were available for post-Stroop Navon analysis. Spectral analysis using the pwelch() function was conducted in MATLAB (The MathWorks, Inc.) using the EEGLAB toolbox (Delorme \& Makeig, 2004). A Hamming window with a window size consisting of 512 data points (i.e., 1.024 seconds) and $50 \%$ overlap allowed for the extraction of average alpha power density at the frontal sites (F3, F4, F7, F8) separately for Navon letter stimuli presented pre-Stroop and post-Stroop Navon manipulation. Similar to resting EEG analysis, a medial frontal asymmetrical index $(\log \mathrm{F} 4-\log \mathrm{F} 3)$ and a lateral frontal asymmetrical index (log F8 - $\log$ F7) were averaged together to create an overall frontal asymmetry score. This resulted in two overall frontal asymmetry scores: one for trials before the Stroop manipulation (pre-Stroop Navon frontal asymmetry) and one for trials after the Stroop manipulation (post-Stroop Navon frontal asymmetry).

\section{Results}

\section{Behavioral results}

All correct Stroop response times were extracted $\left(M_{\text {reject }}=\right.$ $3.76 \%, S D_{\text {reject }}=3.16 \%$ ) and subjected to the same twostage recursive outlier elimination procedure in Experiment $1\left(M_{\text {reject }}=8.41 \%, S D_{\text {reject }}=1.98 \%\right)$. As well, all correct global/local response times (RTs) that were less than $2,000 \mathrm{~ms}$ were extracted $\left(M_{\text {reject }}=2.68 \%, S D_{\text {reject }}=2.45 \%\right)$ and subjected to the same two-stage recursive outlier elimination procedure in Experiment $1\left(M_{\text {reject }}=8.57 \%, S D_{\text {reject }}=\right.$ $1.78 \%$ ). Again, there was a significant positive relationship between attentional breadth before Stroop and after Stroop, $r$ $=.59, p<.001$, and there were large individual differences in attentional breadth and its change.

To investigate whether individual differences in naturally occurring approach motivation predicted changes in individuals' attentional breadth, we correlated changes in attentional breadth and trait BAS scores and found that greater BAS predicted narrowed attention post-Stroop relative to preStroop, but the relationship was nonsignificant, $r=-.15, p=$ .29 . $^{7}$ To further examine the relationship of individuals'

\footnotetext{
${ }^{7}$ Since behavioral task procedures were the same for participants assigned to the incongruent Stroop condition in Experiment 1 and all participants in Experiment 2, we regressed changes in attentional breadth on BAS (centered), BAS by Experiment, and Experiment. BAS significantly predicted narrowing of attentional breadth, $r=-.29, p<.01$, while both the interaction of BAS by Experiment, and the main effect of Experiment were nonsignificant, $p \mathrm{~s}>.14$.
} 
approach motivated tendencies and their change in breadth, a 2 (low vs. high BAS group) $\times 2$ (pre-Stroop Navon vs. postStroop Navon time) mixed-factors ANOVA was performed. ${ }^{8}$ Participants were grouped based on their total BAS scores into either the low BAS group $(M=35.80, S D=3.35)$ or the high BAS group $(M=43.18, S D=3.00)$ by $z$ scoring BAS and separating high and low groups based on whether individuals' centered scores were above or below zero, respectively. The pattern of results replicated those of Experiment 1 (see Fig. 4). There was a significant interaction of approach motivation and pre- versus post-Stroop Navon on attentional breadth, $F(1,61)$ $=4.94, p=.03, \eta^{2}=.08$, where the effect of pre-Stroop versus post-Stroop Navon was opposite for those with low and high BAS scores. There was no significant main effect of approach motivation, $F(1,61)=1.19, p=.28, \eta^{2}=.02$, or pre-Stroop/ post-Stroop Navon, $F(1,61)=.03, p=.87, \eta^{2}<.01$.

Independent-samples $t$ tests showed no significant difference in attentional breadth for low and high BAS groups before they performed the Stroop task, $t(61)=.02, p=.99, d<$ .01 , but the low BAS participants showed significantly greater attentional breadth than the high BAS participants after performing the Stroop task, $t(61)=2.26, p=.03, d=0.57$, suggesting that individuals' attentional breadth differed following the self-control manipulation depending on their approach-motivated tendencies.

There was a significant relationship where greater selfreported difficulty completing the Stroop task was associated with a narrowing of attention, $r=-.34, p<.01$, even over and above BAS scores, partial $r=-.33, p<.01$, replicating the pattern of results in Experiment 1. Further, when changes in attentional breadth were regressed on BAS (centered), selfreported difficulty (centered) and the interaction of BAS by self-reported difficulty, the BAS by self-reported difficulty interaction was nonsignificant, $t(59)=-.29, p=.77$.

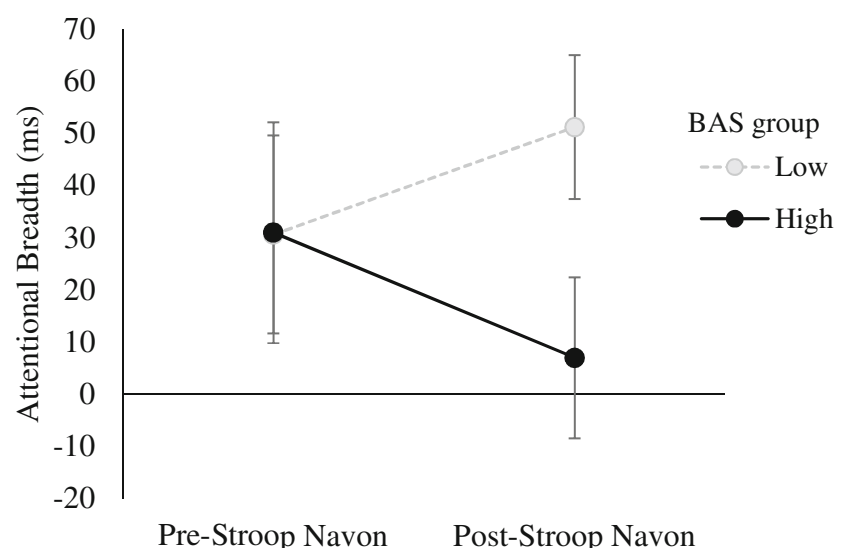

Fig. 4 Average attentional breadth (local RT - global RT) for Navon stimuli as a function of individuals' BAS group and whether the stimuli were presented before or after completing Stroop. Negative values reflect greater local focus, while positive values reflect greater global focus. Error bars represent \pm 1 SEM

\section{Frontal asymmetry}

Individuals' average asymmetry measures were highly consistent across resting, pre-Stroop Navon and post-Stroop Navon blocks, $r \mathrm{~s}>.78$, which is in line with the characterization of frontal asymmetry as a stable individual difference measure (Tomarken, Davidson, Wheeler, \& Doss, 1992a).

Individual differences in BAS scores did not relate to individual differences in frontal asymmetry during the experiment, either while resting or completing the Navon task, $r \mathrm{~s}$ $<.12, p s>.05$. Furthermore, the relationship between BAS and individuals' frontal asymmetry remained nonsignificant when controlling for variables that have previously been shown to influence frontal asymmetry: age, gender, and the time of day, $p$ s $>$.10.Individuals' resting frontal asymmetry did not relate with any attentional breadth measures (i.e., change or pre-Stroop and post-Stroop Navon), $p \mathrm{~s}>.65$.

To examine whether individuals' frontal asymmetry changed after exercising self-control, and whether this was modulated by their approach motivation, a 2 (low vs. high BAS group) $\times 2$ (pre-Stroop Navon vs. post-Stroop Navon time) mixed-factors ANOVA was performed on frontal asymmetry values acquired during the Navon task blocks (see Fig. 5). Post-Stroop Navon frontal asymmetry was almost significantly different from preStroop Navon frontal asymmetry, $F(1,52)=3.57, p=.06, \eta^{2}=$ .06 , and there was no significant main effect of BAS group, $F(1$, $52)=.24, p=.62, \eta^{2}<.01$. Contrary to our hypothesis, there was no significant interaction of BAS and pre-Stroop/postStroop Navon on individuals' frontal asymmetry, $F(1,52)=$ $.20, p=.66, \eta^{2}<.01$, in that both low and high BAS participants showed small, nonsignificant, increases in left relative frontal cortical activity from pre-Stroop to post-Stroop Navon.

General discussionThe purpose of this study was to examine how self-control could influence attentional breadth differently depending on individuals' levels of BAS and explore frontal asymmetry as a possible mechanism. This is an important question, as human beings often have to constrain and inhibit easy or predominant thoughts and behaviors in everyday life, and this could affect their subsequent cognitive processing. While resisting eating the delicious piece of cake may be beneficial for one's health in the long term, what are the consequences of this self-control for their cognitive processing in the short term?

In two studies, we looked at attentional breadth before and after a self-control task while measuring BAS, and in Study 2 we also measured frontal asymmetry, both at rest and before each Navon trial. Although previous work has investigated various combinations of self-control, BAS, attentional breadth, resting frontal asymmetry, and pretrial frontal asymmetry, Experiment 2 is the first study to combine all of these measure into a single study to examine whether the changes in attentional breadth as a function of self-control and BAS are related to resting asymmetrical frontal activity or its change in frontal asymmetry across trials. 


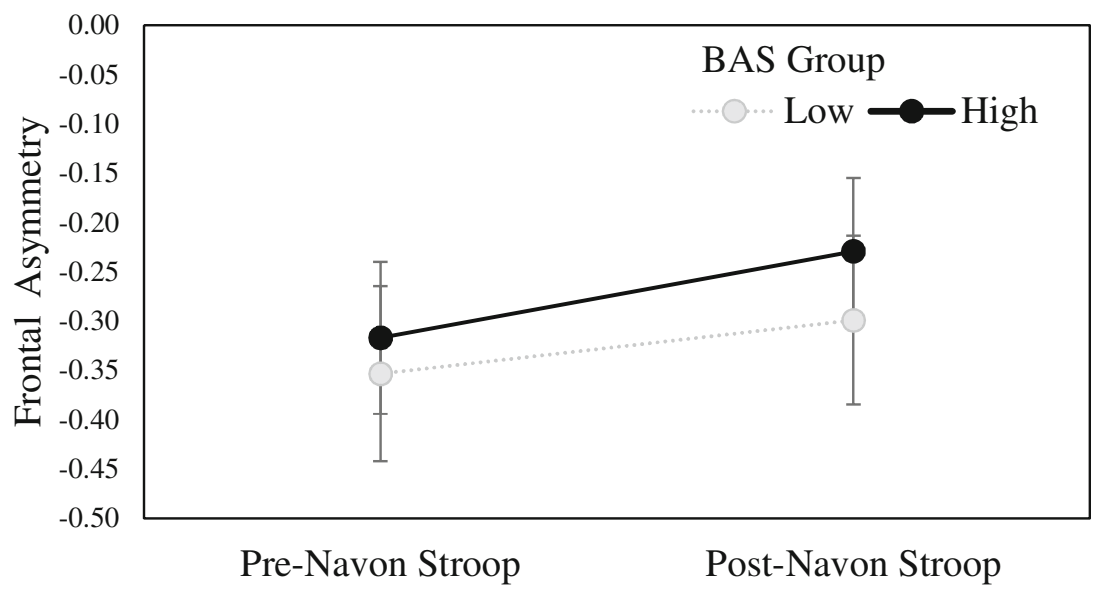

Fig. 5 Average frontal asymmetry as a function of individuals' BAS group and whether measures were taken before or after completing Stroop. Negative values reflect greater alpha activity in the left frontal

electrode sites, most likely suggesting greater average cortical activity in the right prefrontal areas. Error bars represent \pm 1 SEM

There were three main findings: (1) Self-control influenced attentional breadth differently depending on BAS where the self-control manipulation reduced attentional breadth only for the high BAS individuals; (2) narrowed attentional breadth related to greater subjective reports of difficulty while exercising self-control, and this was independent of the BAS effect on attentional breadth after self-control; and (3) BAS, attentional breadth, and changes in attentional breadth, were found to be unrelated to individual differences in resting or pretrial frontal asymmetry, or changes in pretrial alpha asymmetry pre-Stroop to post-Stroop Navon.

\section{Approach and attentional breadth}

Modulations in breadth of attention can serve a purpose in helping humans interact appropriately with the environment. For example, attentional breadth has been shown to narrow after viewing approach-motivating stimuli (e.g., the tasty treat when it has been hours since last eating), and this may allow for individuals to "keep their eyes on the prize" and pursue their goals (Gable \& Harmon-Jones, 2011; Harmon-Jones \& Gable, 2009). Furthermore, individuals differ in their tendencies to approach in everyday life, and individuals with greater tendencies show more narrowed attentional breadth when viewing approach-motivating images in comparison to individuals with lesser tendencies (Gable \& Harmon-Jones, 2008).

The results from this study largely support the hypothesis that there are attentional changes, or shifts, that occur after exercising self-control, and that these attentional changes are largely dependent on individuals' levels of naturally occurring or trait levels of approach motivation such that exerting selfcontrol promotes narrowed attention for those high in BAS. The narrowed attention for high BAS individuals after engaging in self-control would be generally consistent with the process model of self-control (Inzlicht \& Schmeichel, 2012;
Inzlicht et al., 2014). This model postulates that exerting self-control causes shifts in attention and motivation toward approaching more reward-seeking and pleasurable behaviors. Evidence to support this model includes findings that selfcontrol can lead to greater reported optimism in highapproach-motivated individuals (Crowell et al., 2014), facilitated perception of reward-like symbols in comparison to nonreward-like symbols (Schmeichel et al., 2010), and muted error monitoring (via decreased error-related negativity amplitudes) during incorrect trials by individuals with greater left frontal activity at rest (Nash et al., 2012).

If self-control does lead to greater approach motivation, as the process model predicts, then one might expect the increased approach state following self-control to lead to a narrowing of attention on global/local tasks, especially in those individuals with high dispositional approach motivation (e.g., Gable \& Harmon-Jones, 2008). The present results support this hypothesis in that high BAS individuals showed narrowed attentional breadth after exercising self-control, but not after completing a non-self-control task.

As noted above, Crowell et al. (2014) used a different attentional breadth measure, and a different self-control task, and found that attentional breadth was significantly more broad for high-approach individuals after exercising self-control, which is opposite to the findings reported here. They also claimed that their results provided evidence that self-control increased approach motivation for high BAS individuals, although this conclusion fits their findings less well given the well-replicated relationship between attentional narrowing and greater approach motivation (e.g., Domachowska et al., 2016; Gable \& Harmon-Jones, 2008; Gable, Mechin, \& Neal, 2016; Harmon-Jones \& Gable, 2009; Harmon-Jones, Price, \& Gable, 2012; Hicks, Friedman, Gable, \& Davis, 2012; Juergensen \& Demaree, 2015; Liu, Wang, Quan, \& Li, 2017; Liu, Zhang, Zhou, \& Wang, 2014). Some findings in the literature suggest that approach motivation can result in 
broadened attentional breadth in contexts other than viewing images of appetitive stimuli (e.g., see Förster, Friedman, Ozelsel, \& Denzler, 2006; Friedman \& Förster, 2010). Indeed, Crowell and colleagues suggested that their selfcontrol manipulation induced a low-intensity approach-motivational state in contrast to the high-intensity approach-motivational state induced when viewing appetitive images, and this resulted in greater breadth for high BAS individuals. One possible explanation for the differences in findings is that the self-control task used here (incongruent Stroop) created a different context than that of the self-control task used by Crowell et al. (2014), and that this lead to a different role for approach motivation in the two studies.

Another explanation for the different results found here could be because of differences in the attentional breadth task used, as weak correlations have been previously reported between various attentional breadth measures, suggesting that they tap into distinct aspects of global/local processing (Chamberlain, Van de Hallen, Huygelier, Van der Cruys, \& Wagemans, 2017; Dale \& Arnell, 2013). However, it is currently unclear why eliciting greater self-control could potentially broaden attention for high-approach-motivated individuals when completing one attentional breadth task, but narrow attention when using a different attentional breadth measure, although other contextual factors could be considered in future research (e.g., whether appetitive stimuli are presented following self-control). Further, many elements of the incongruent Stroop task may have played a role in affecting attentional breadth (e.g., fatigue, signaling of conflict, increases in heart rate and arousal, changes in stress, changes in affect; Fröber, Stürmer, Frömer, \& Dreisbach, 2017; Renaud \& Blondin, 1997). Although a strong argument could be made for the inclusion of these factors in the construct of self-control, it is unclear whether any of these effects on their own had a disproportionate influence on the change in attentional breadth or how these may differ from the controlled writing self-control task used by Crowell et al. (2014). Further, self-control is a complex construct, and although Stroop is a popular and validated measure of self-control (e.g., see Duckworth \& Kern, 2011), future research would benefit from examining multiple measures of self-control and examining how they influence attentional breadth.

Individuals show reliable individual differences in their global/local bias (Dale \& Arnell, 2013). This study is the first to look at how attentional breadth changes before and after self-control, thereby accounting for individuals' baseline global/local biases. In contrast, Crowell et al. (2014) randomly assigned individuals to high or low self-control conditions and measured attentional breadth only after the self-control manipulation, meaning the large individual differences were not removed. However, regardless of the reason for the different results across the two studies, the replication of the behavioral results across Experiments 1 and 2 give us confidence that the pattern of results reported here is reliable, and the present behavioral results fit the pattern one would expect based on previous links between attentional breadth, self-control, and approach motivation.

\section{Difficulty with self-control}

The self-control required during the Stroop task was indirectly measured via the self-reported difficulty of the Stroop task. Asking participants to rate their subjective experience while completing the Stroop task may offer an approximate estimate of how much self-control was required to perform the Stroop task. Interestingly, the extent to which participants found the self-control task to be difficult was largely independent of their approach-motivated tendencies and independently influenced the change in attentional breadth in the same direction, where greater difficulty during the self-control task predicted a narrower attentional focus and less propensity to see the "bigger picture," or the "forest for the trees." Accordingly, there was no relationship between difficulty and attentional breadth for individuals that did the congruent Stroop task in Experiment 1, where there was no need to exercise self-control. Individuals who found the task to be easier, either because they were in the congruent Stroop condition or they excelled in the incongruent Stroop condition, may have been more likely to experience a more positive, low-intensity mood, which is often associated with a more diffuse, broad focus (Fredrickson \& Branigan, 2005; Gasper \& Clore, 2002). In contrast, greater difficulty could be associated with the urgency to utilize resources to act upon the immediate environment and greater attentional investment, as greater effort may have been necessary for these individuals to complete the task.

The results from this study suggest that the difficulty completing the task and propensity for individuals to approach are two mechanisms that affect attentional breadth in the same direction. They are both associated with narrower attentional focus, but are largely independent from one another. This suggests that it is not simply that high-approach-motivated individuals found the task more difficult, and that the greater challenge experienced by these individuals accounted for changes in attentional breadth; there seems to be at least two mechanisms influencing attentional breadth following selfcontrol. Research examining the aftereffects of self-control on attentional breadth should focus on both individuals' approach motivation and difficulty while completing the selfcontrol task.

\section{The role of frontal asymmetry}

Frontal asymmetry measures showed high consistency with each other across time (pre-Stroop vs. post-Stroop Navon), task (resting vs. pretrial), and electrode location (medial vs. lateral), which supports the view that frontal asymmetry is a 
reliable individual difference measure (Tomarken, Davidson, Wheeler, \& Doss, 1992a). The results here provide some support for the process model if one were to examine the change in frontal asymmetry over all participants, in that the marginally significant greater left relative frontal activity following the self-control task could be due to greater approach motivation as posited by the process model. However, in the current study, frontal asymmetry and its changes were unrelated to attentional breadth and its changes after self-control. Because the behavioral change was present without the frontal asymmetry change, the present results provide evidence that the behavioral change in attentional breadth for high BAS participants following self-control does not rely on changes in frontal activation patterns, and that an increase in left frontal activation is not the mechanism that underlies this effect.

Why might previous studies have observed frontal asymmetry changes after self-control that differ depending on individuals BAS, even though no such changes were observed here? In the present study, frontal asymmetry was measured in the absence of emotionally salient stimuli, such as positive or negative images, allowing us to examine whether selfcontrol can influence frontal asymmetry without emotional picture viewing. Schmeichel et al. (2016) found that increased left frontal activity occurred only for individuals that were high in approach-motivated tendencies and had subsequently viewed positive images after exercising self-control, suggesting the combination of high approach stimuli and high approach-oriented individuals was needed (see also HarmonJones, Lueck, Fearn, \& Harmon-Jones, 2013, for further evidence and discussion of this point). Greater left frontal activity was not found when these individuals had viewed neutral images, suggesting that self-control on its own was not sufficient to examine the relationship between approach motivation and frontal asymmetry. The finding of the null relationships between asymmetry and attentional breadth in the present study after self-control, even in those with high BAS, is therefore consistent with the null results from the neutral picture condition of Schmeichel et al. (2016).

There may not have been a strong enough emotional challenge in the present study to elicit significant changes in frontal asymmetry that differ between the two BAS groups. It is possible that viewing emotional images after exercising selfcontrol may influence individuals' attentional breadth beyond the effect of exercising self-control alone, and that the effect of viewing the emotional images after exercising self-control is what lead to greater relative left frontal activity for high BAS individuals, as reported by Schmeichel et al. (2016). Indeed, frontal asymmetry has been proposed to more strongly associate with approach motivation when there are explicit cues of incentive anticipation presented, and these cues were not included in the current design (Harmon-Jones \& Gable, 2018).

Another finding that replicated previous work was that individual differences in approach-motivated tendencies, measured by people's responses to the well-validated BIS/ BAS questionnaire, did not relate to individual differences in resting frontal asymmetry. This is consistent with some findings in the literature (Boksem et al., 2012; Neal \& Gable, 2017; Wacker et al., 2010) that have also shown no relationship between BAS scores and frontal asymmetry, but is inconsistent with others (Coan \& Allen, 2003; Harmon-Jones \& Allen, 1997) that have shown associations between greater relative left frontal activation and higher BAS scores. However, frontal asymmetry did indeed show qualities of a stable individual difference measure here and has been shown in previous work to relate to approach motivation when there is a stronger emotional experience present (Wacker et al., 2010). In future experiments, if we were to present an appetitive picture before each Navon trial, we may then see a relationship between frontal activation patterns and BAS, and see frontal activation shift to the left hemisphere in high BAS individuals after self-control. However, the fact that we did not see this shift in the present study, yet high BAS individuals still showed the attentional narrowing after self-control, means that these results strongly suggest that changes in frontal activation patterns do not underlie the changes in attentional breadth observed after self-control, for either high or low BAS individuals.

\section{Conclusion}

The results of these two studies show that exercising selfcontrol influences attentional breadth of high and low approach-motivated individuals differently where high approach individuals narrow attention following self-control. Furthermore, these changes in attentional breadth are unrelated to asymmetrical frontal cortical activity or its change, suggesting that frontal asymmetry is not the mechanism that underlies changes in attentional breadth with self-control. This is the first study to determine that changes in attentional breadth following self-control are not associated with individual differences in asymmetrical frontal cortical activity.

This is also the first study to show that greater self-reported difficulty completing the self-control task was also associated with narrowing of attention post-self-control relative to preself-control, and the influence of difficulty on changes in attentional breadth was largely independent of the effect of approach-motivated tendencies.

The results provide some support for the process model of self-control (Inzlicht \& Schmeichel, 2012; Inzlicht et al., 2014) in that exercising self-control can modulate attentional breadth depending on individual differences in motivated tendencies. Induced high approach states have been previously found to promote narrow attentional processing (e.g., Gable \& Harmon-Jones, 2008). Therefore, finding that high approach individuals become more narrowly focused after self-control is consistent with the process model's central premise that 
high approach individuals move toward an approach state after exercising effortful self-control. As well, the increase in relative left frontal activity following the completion of selfcontrol could indicate increased approach motivation as the model predicts, but it is unclear why individuals' approachmotivated tendencies did not moderate the increase in relative left activity, or why greater relative left activity did not associate with narrowed attentional breadth. Finally, the process model could easily accommodate the findings that ratings of Stroop difficulty would predict changes in attentional breadth if it assumed that the more effortful the self-control, the greater the move toward an approach state overall.

Acknowledgements The work was supported by a Canadian Graduate Scholarship from the Natural Sciences and Engineering Research Council of Canada (NSERC) to the first author, and by a grant from NSERC to the second author. This work was presented in October 2018 at the annual meeting of the Vision Sciences Society meeting in St. Pete Beach, United States of America..

\section{References}

Allen, J. J. B., Coan, J. A., \& Nazarian, M. (2004). Issues and assumptions on the road from raw signals to metrics of frontal EEG asymmetry in emotion. Biological Psychology, 67(1/2), 183-218. https:// doi.org/10.1016/j.biopsycho.2004.03.007

Boksem, M. A. S., Kostermans, E., Tops, M., \& De Cremer, D. D. (2012). Individual differences in asymmetric resting-state frontal cortical activity modulate ERPS and performance in a global-local attention task. Federation of European Psychophysiology Societies, 26(2), 51-62. https://doi.org/10.1027/0269-8803/a000067

Botvinick, M. M. (2007). Conflict monitoring and decision making: Reconciling two perspectives on anterior cingulate function. Cognitive, Affective, \& Behavioral Neuroscience, 7(4), 356-366. https://doi.org/10.3758/CABN.7.4.356

Carver, C. S., \& White, T. L. (1994). Behavioral inhibition, behavioral activation, and affective responses to impending reward and punishment: The BIS/BAS Scales. Journal of Personality and Social Psychology, 67(2), 319-333. https://doi.org/10.1037/0022-3514. 67.2.319

Chamberlain, R., Van der Hallen, R., Huygelier, H., Van de Cruys, S., \& Wagemans, J. (2017). Local-global processing bias is not a unitary individual difference in visual processing. Vision Research, 141, 247-257. https://doi.org/10.1016/j.visres.2017.01.008

Coan, J. A., \& Allen, J. J. B. (2003). Frontal EEG asymmetry and the behavioral activation and inhibition systems. Psychophysiology, 40(1), 106-114. https://doi.org/10.1111/1469-8986.00011

Coan, J. A., \& Allen, J. J. B. (2004). Frontal EEG asymmetry as a moderator and mediator of emotion. Biological Psychology, 67, 7-49. https://doi.org/10.1016/j.biopsycho.2004.03.002

Crowell, A., Kelley, N. J., \& Schmeichel, B. J. (2014). Trait approach motivation moderates the aftereffects of self-control. Frontiers in Psychology, 5(112), 1-10. https://doi.org/10.3389/fpsyg.2014. 01112

Dale, G., \& Arnell, K. M. (2011). An investigation of the reliability and relationships among global-local processing measures. Journal of Vision, 11(11), 149-149. https://doi.org/10.1167/11.11.149

Dale, G., \& Arnell, K. M. (2013). Investigating the stability of and relationships among global/local processing measures. Attention,
Perception, \& Psychophysics, 75, 394-406. https://doi.org/10. 3758/s13414-012-0416-7

Dale, G., \& Arnell, K. M. (2014). Lost in the forest, stuck in the trees: Dispositional global/local bias is resistant to exposure to high and low spatial frequencies. PLOS ONE, (7), https://doi.org/10.1371/ journal.pone. 0098625

De Pascalis, V., Cozzuto, G., Caprara, G. V., \& Alessandri, G. (2013). Relations among EEG-alpha asymmetry, BIS/BAS, and dispositional optimism. Biological Psychology, 94(1), 198-209. https://doi.org/ 10.1016/j.biopsycho.2013.05.016

Delorme, A., \& Makeig, S. (2004). EEGLAB: An open source toolbox for analysis of single-trial EEG dynamics including independent component anlaysis. Journal of Neuroscience Methods, 134, 9-21. https://doi.org/10.1016/j.jneumeth.2003.10.009

Diamond, A. (2013). Executive functions. Annual Review of Psychology, 64(1), 135-168. https://doi.org/10.1146/annurev-psych-113011143750

Domachowska, I., Heitmann, C., Deutsch, R., Goschke, T., Scherbaum, S., \& Bolte, A. 2016. Approach-motivated positive affect reduces breadth of attention: Registered replication report of Gable and Harmon-Jones (2008). Journal of Experimental Social Psychology, 67(Special Issue: Confirmatory), 50-56. https://doi.org/10.1016/j. jesp.2015.09.003

Duckworth, A. L., \& Kern, M. L. (2011). A meta-analysis of the convergent validity of self-control measures. Journal of Research in Personality, 45(3), 259-268. https://doi.org/10.1016/j.jrp.2011.02. 004.A

Düsing, R., Tops, M., Radtke, E. L., Kuhl, J., \& Quirin, M. (2016). Relative frontal brain asymmetry and cortisol release after social stress: The role of action orientation. Biological Psychology, 115, 86-93. https://doi.org/10.1016/j.biopsycho.2016.01.012

Fecteau, S., Knoch, D., Fregni, F., Sultani, N., Boggio, P., \& PascualLeone, A. (2007). Diminishing risk-taking behavior by modulating activity in the prefrontal cortex: A direct current stimulation study. Journal of Neuroscience, 27(46), 12500-12505. https://doi.org/10. 1523/JNEUROSCI.3283-07.2007

Förster, J., Friedman, R. S., Ozelsel, A., \& Denzler, M. (2006). Enactment of approach and avoidance behavior influences the scope of perceptual and conceptual attention. Journal of Experimental Social Psychology, 42, 133-146. https://doi.org/10.1016/j.jesp.2005.02.004

Fredrickson, B. L., \& Branigan, C. (2005). Positive emotions broaden the scope of attention and thought-action repertoires. Cognition and Emotion, 19(3), 313-332. https://doi.org/10.1080/ 02699930441000238

Fregni, F., Orsati, F., Pedrosa, W., Fecteau, S., Tome, F. A. M., Nitsche, M. A., . . Boggio, P. S. (2008). Transcranial direct current stimulation of the prefrontal cortex modulates the desire for specific foods. Appetite, 51(1), 34-41. https://doi.org/10.1016/j.appet.2007.09.016

Friedman, R. S., \& Förster, J. (2010). Implicit affective cues and attentional tuning: An integrative review. Psychological Bulletin, 136, 875-893. https://doi.org/10.1037/a0020495

Fröber, K., Stürmer, B., Frömer, R., \& Dreisbach, G. (2017). The role of affective evaluation in conflict adaptation: An LRP study. Brain and Cognition, 116(May), 9-16. https://doi.org/10.1016/j.bandc.2017. 05.003

Gable, P. A., \& Harmon-Jones, E. (2008). Approach-motivated positive affect reduces breadth of attention. Psychological Science, 19(5), 476-482. https://doi.org/10.1111/j.1467-9280.2008.02112.x

Gable, P. A., \& Harmon-Jones, E. (2011). Attentional consequences of pregoal and postgoal positive affects. Emotion, 11(6), 1358-1367. https://doi.org/10.1037/a0025611

Gable, P. A., Mechin, N. C., Hicks, J. A., \& Adams, D. L. (2015). Supervisory control system and frontal asymmetry: Neurophysiological traits of emotion-based impulsivity. Social Cognitive and Affective Neuroscience, 10(10), 1310-1315. https:// doi.org/10.1093/scan/nsv017 
Gable, P. A., Mechin, N. C., \& Neal, L. B. (2016). Booze cues and attentional narrowing: Neural correlates of virtual alcohol myopia. Psychology of Addictive Behaviors, 30(3), 377-382. https://doi.org/ 10.1037/adb0000130

Gable, P. A., Poole, B. D., \& Cook, M. S. (2013). Asymmetrical hemisphere activation enhances global-local processing. Brain and Cognition, 83(3), 337-341. https://doi.org/10.1016/j.bandc.2013. 09.012

Gasper, K., \& Clore, G. L. (2002). Attending to the big picture: Mood and global versus local processing of visual information. Psychological Science, 13(1), 34-40. https://doi.org/10.1111/1467-9280.00406

Grimshaw, G. M., Foster, J. J., \& Corballis, P. M. (2014). Frontal and parietal EEG asymmetries interact to predict attentional bias to threat. Brain and Cognition, 90, 76-86. https://doi.org/10.1016/j. bandc.2014.06.008

Harmon-Jones, E., \& Allen, J. J. B. (1997). Behavioral activation sensitivity and resting frontal EEG asymmetry: Covariation of putative indicators related to risk for mood disorders. Journal of Abnormal Psychology, 106(1), 159-163. https://doi.org/10.1037/0021-843X. 106.1.159

Harmon-Jones, E., \& Allen, J. J. B. (1998). Anger and frontal brain activity: EEG asymmetry consistent with approach motivation despite negative affective valence. Journal of Personality and Social Psychology, 74(5), 1310-1316. https://doi.org/10.1037/0022-3514. 74.5.1310

Harmon-Jones, E., \& Gable, P. A. (2009). Neural activity underlying the effect of approach-motivated positive affect on narrowed attention. Psychological Science, 20(4), 406-409. https://doi.org/10.1111/j. 1467-9280.2009.02302.x

Harmon-Jones, E., \& Gable, P. A. (2018). On the role of asymmetric frontal cortical activity in approach and withdrawal motivation: An updated review of the evidence. Psychophysiology, 55(1. https://doi. org/10.1111/psyp.12879

Harmon-Jones, E., Lueck, L., Fearn, M., \& Harmon-Jones, C. (2013). The effect of personal relevance action and approach-related action expectation on relative left frontal cortical activity. Psychological Science, 17(5), 434-440. https://doi.org/10.1111/j.1467-9280.2006.01724.x

Harmon-Jones, E., Price, T. F., \& Gable, P. A. (2012). The influence of affective states on cognitive broadening/narrowing: Considering the importance of motivational intensity. Social and Personality Psychology Compass, 6(4), 314-327. https://doi.org/10.3389/fnint. 2012.00073

Hicks, J. A., Friedman, R. S., Gable, P. A., \& Davis, W. E. (2012). Interactive effects of approach motivational intensity and alcohol cues on the scope of perceptual attention. Addiction, 107(6), 10741080. https://doi.org/10.1111/j.1360-0443.2012.03781.x

Hofmann, W., Vohs, K. D., \& Baumeister, R. F. (2012). What people desire, feel conflicted about, and try to resist in everyday life. Psychological Science, 23(6), 582-588. https://doi.org/10.1177/ 0956797612437426

Hughes, D. M., Yates, M. J., Morton, E. E., \& Smillie, L. D. (2014). Asymmetric frontal cortical activity predicts effort expenditure for reward. Social Cognitive and Affective Neuroscience, 10(7), 10151019. https://doi.org/10.1093/scan/nsu149

Inzlicht, M., \& Schmeichel, B. J. (2012). What is ego depletion? Toward a mechanistic revision of the resource model of self-control. Perspectives on Psychological Science, 7(5), 450-463. https://doi. org/10.1177/1745691612454134

Inzlicht, M., Schmeichel, B. J., \& Macrae, C. N. (2014). Why self-control seems (but may not be) limited. Trends in Cognitive Sciences, 18(3), 127-133. https://doi.org/10.1016/j.tics.2013.12.009

Juergensen, J., \& Demaree, H. A. (2015). Approach-motivated positive affect and emotion regulation alter global-local focus and food choice. Motivation and Emotion, 39, 580-588. https://doi.org/10. 1007/s11031-015-9472-3
Kelley, N. J., Hortensius, R., Schutter, D. J. L. G., \& Harmon-Jones, E. (2017). The relationship of approach/avoidance motivation and asymmetric frontal cortical activity: A review of studies manipulating frontal asymmetry. International Journal of Psychophysiology, 119, 19-30. https://doi.org/10.1016/j.ijpsycho.2017.03.001

Kimchi, R. (1992). Primacy of wholistic processing and global/local paradigm: A critical review. Psychological Bulletin, 112(1), 24-38. https://doi.org/10.1037/0033-2909.112.1.24

Kimchi, R., \& Palmer, S. E. (1982). Form and texture in hierarchically constructed patterns. Journal of Experimental Psychology: Human Perception and Performance, 8(4), 521-35. https://doi.org/10.1037/ 0096-1523.8.4.521

Kinchla, R. A., \& Wolfe, J. M. (1979). The order of visual processing: "Top-down," "bottom-up," or "middle-out". Perception \& Psychophysics, 25(3), 225-231. https://doi.org/10.3758/ BF03202991

Liu, L., Zhang, G., Zhou, R., \& Wang, Z. (2014). Motivational intensity modulates attentional scope: Evidence from behavioral and ERP studies, Experimental Brain Research, 232, 3291-3300, https:// doi.org/10.1007/s00221-014-4014-x

Liu, Y., Wang, Z., Quan, S., \& Li, M. (2017). The effect of positive affect on conflict resolution: Modulated by approach-motivational intensity. Cognition and Emotion, 31(1), 69-82. https://doi.org/10.1080/ 02699931.2015.1081874

Nash, K., Inzlicht, M., \& McGregor, I. (2012). Approach-related left prefrontal EEG asymmetry predicts muted error-related negativity. Biological Psychology, 91(1), 96-102. https://doi.org/10.1016/j. biopsycho.2012.05.005

Navon, D. (1977). Forest before trees: The precedence of global features in visual perception. Cognitive Psychology, 9(3), 353-383. https:// doi.org/10.1016/0010-0285(77)90012-3

Neal, L. B., \& Gable, P. A. (2017). Regulatory control and impulsivity relate to resting frontal activity. Social Cognitive and Affective Neuroscience, 12(9), 1377-1383. https://doi.org/10.1093/scan/ nsx080

Paquet, L., \& Merikle, P. M. (1984). Global precedence: The effect of exposure duration. Canadian Journal of Psychology, 38(1), 45-53. https://doi.org/10.1037/h0080783

Pérez-Edgar, K., Kujawa, A., Nelson, S. K., Cole, C., \& Zapp, D. J. (2013). The relation between electroencephalogram asymmetry and attention biases to threat at baseline and under stress. Brain and Cognition, 82, 337-343. https://doi.org/10.1016/j.bandc.2013. 05.009

Pizzagalli, D. A., Sherwood, R. J., Henriques, J. B., \& Davidson, R. J. (2005). Frontal brain asymmetry and reward responsiveness. Psychological Science, 16(10), 805-813. https://doi.org/10.1111/j. 1467-9280.2005.01618.x

Price, T. F., \& Harmon-Jones, E. (2010). The effect of embodied emotive states on cognitive categorization. Emotion, 10(6), 934-938. https:// doi.org/10.1037/a0019809

Quaedflieg, C. W. E. M., Meyer, T., Smulders, F. T. Y., \& Smeets, T. (2015). The functional role of individual-alpha based frontal asymmetry in stress responding. Biological Psychology, 104, 75-81. https://doi.org/10.1016/j.biopsycho.2014.11.014

Renaud, P., \& Blondin, J.-P. (1997). The stress of Stroop performance: Physiological and emotional responses to color-word interference, task pacing, and pacing speed. International Journal of Psychophysiology, 27, 87-97. https://doi.org/10.1016/S01678760(97)00049-4

Schmeichel, B. J., Crowell, A., \& Harmon-Jones, E. (2016). Exercising self-control increases relative left frontal cortical activation. Social Cognitive and Affective Neuroscience, 11(2), 282-288. https://doi. org $/ 10.1093 / \mathrm{scan} / \mathrm{nsv} 112$

Schmeichel, B. J., Harmon-Jones, C., \& Harmon-Jones, E. (2010). Exercising self-control increases approach motivation. Journal of 
Personality and Social Psychology, 99(1), 162-173. https://doi.org/ 10.1037/a0019797

Shields, G. S., \& Moons, W. G. (2016). Avoidance-related EEG asymmetry predicts circulating interleukin-6. Emotion, 16(2), 150-154. https://doi.org/10.1037/emo0000120

Spielberg, J. M., Miller, G. A., Warren, S. L., Engels, A. S., Crocker, L. D., Sutton, B. P., \& Heller, W. (2012). Trait motivation moderates neural activation associated with goal pursuit. Cognitive, Affective, \& Behavioral Neuroscience, 12(2), 308-22. https://doi.org/10.3758/ s13415-012-0088-8

Spielberg, J. M., Stewart, J. L., Levin, R. L., Miller, G. A., \& Heller, W. (2010). Prefrontal cortex, emotion, and approach/ withdrawl motivation. Social and Personality Psychology Compass, 2(1), 135-153. https://doi.org/10.1111/j.1751-9004. 2007.00064.x.Prefrontal

Stroop, J. R. (1935). Studies of interference in serial verbal reactions. Journal of Experimental Psychology, 18(6), 643-662. https://doi. org $/ 10.1037 / \mathrm{h} 0054651$
Tomarken, A. J., Davidson, R. J., Wheeler, R. E., \& Doss, R. C. (1992a). Individual differences in anterior brain asymmetry and fundamental dimensions of emotion. Journal of Personality and Social Psychology, 62(4), 676-687. https://doi.org/10.1037/0022-3514.62.4.676

Tomarken, A. J., Davidson, R. J., Wheeler, R. E., \& Kinney, L. (1992b). Psychometric properties of resting anterior EEG asymmetry: Temporal stability and internal consistency. Psychophysiology. https://doi.org/10.1111/j.1469-8986.1992.tb02034.x

Wacker, J., Chavanon, M. L., \& Stemmler, G. (2010). Resting EEG signatures of agentic extraversion: New results and meta-analytic integration. Journal of Research in Personality, 44(2), 167-179. https://doi.org/10.1016/j.jrp.2009.12.004

Wagner, D. D., Altman, M., Boswell, R. G., Kelley, W. M., \& Heatherton, T. F. (2013). Self-regulatory depletion enhances neural responses to rewards and impairs top-down control. Psychological Science, 24(11), 2262-2271. https://doi.org/10.1177/0956797613492985 